\title{
Optical and morphological properties of porous diamond-like-carbon films deposited by magnetron sputtering
}

\author{
M.P.M.A. Baroni ${ }^{\mathrm{a}, *}$, M. Ventura Conceição ${ }^{\mathrm{b}}$, R.R. Rosa ${ }^{\mathrm{a}}$, C. Persson ${ }^{\mathrm{c}}$, H. Arwin ${ }^{\mathrm{d}}$, \\ E.F. da Silva Jr. ${ }^{\text {e, L.S. Roman }}{ }^{\mathrm{f}}$, O. Nakamura ${ }^{\mathrm{b}}$, I. Pepe ${ }^{\mathrm{b}}$, A. Ferreira da Silva ${ }^{\mathrm{b}}$ \\ a Núcleo de Simulação e Análise de Sistemas Complexos, Laboratório Associado de Computação e Matemática Aplicada, \\ Instituto Nacional de Pesquisas Espaciais (INPE), 12201-970 São José dos Campos, SP, Brazil \\ ${ }^{\mathrm{b}}$ Grupo de Física Básica e Aplicada em Materiais Semicondutores, Laboratório de Propriedades Ópticas, Instituto de Física, \\ Universidade Federal da Bahia, Campus Ondina, 40170-290 Salvador, BA, Brazil \\ ${ }^{\mathrm{c}}$ Applied Materials Physics, Department of Materials Science and Engineering, Royal Institute of Technology, SE-100 44 Stockholm, Sweden \\ d Department of Physics, Linköping University, SE 58183 Linköping, Sweden \\ e Departamento de Física, Universidade Federal de Pernambuco, 50670901 Recife, PE, Brazil \\ ${ }^{\mathrm{f}}$ Grupo de Dispositivos Nanoestruturados, Departamento de Física, Universidade Federal do Paraná, 81531-990 Curitiba, PR, Brazil
}

Available online 8 August 2006

\begin{abstract}
Porous diamond-like-carbon (PDLC) thin films obtained on silicon substrate by DC low energy magnetron sputtering have been investigated by photoluminescence, transmission and reflection spectroscopy, photoacoustic and spectroscopic ellipsometry. The absorption features observed for these films show similarities with those of porous silicon (PS) as well as in the performed gradient structural pattern classification of the SFM porosity, by means of the computational GPA-flyby environment on PS and PDLC samples. The dielectric function is also calculated for the bulk diamond-like carbon using the full-potential linearized augmented plane wave method within the framework of local density approximation to density functional theory. From the measurement a low real dielectric constant of about 4.5 at $0.8 \mathrm{eV}$ was found whereas the calculated $e_{1}(0)$ for the bulk diamond has a value of 5.5.
\end{abstract}

(C) 2006 Elsevier B.V. All rights reserved.

PACS: 61.43.Dq; 73.61.-r; 78.20.-e; 78.20.Bh

Keywords: Modeling and simulation; Optical properties; Thermal properties

\section{Introduction}

Porous diamond-like-carbon (PDLC) films have many attractive properties which can deal with potential applications. Besides mechanical and tribological characteristics, electrical and optical properties can, also, deserve special attention $[1,2]$. More specifically, surface porosity in different kinds of materials exhibit very interesting optical effect related to porous density and size, suggesting new applications [3].

\footnotetext{
* Corresponding author. Tel.: +55 12 39456368; fax: +55 1239456375 . E-mail address: mariana@lac.inpe.br (M.P.M.A. Baroni).
}

PDLC obtained by low energy magnetron sputtering on silicon substrate has been investigated by photoluminescence, transmission and reflection spectroscopy, spectroscopic ellipsometry and photoacoustic. For the latter the thermal diffusivity are investigated. In order to obtain the value of the bulk diamond-like carbon (BDLC), the calculation of the dielectric functions using the full-potential linearized augmented plane wave (FPLAPW) method, within the framework of local density approximation (LDA) to density functional theory, is performed [4].

Scanning force microscopy (SFM) and absorption measurements have revealed an intriguing similarity between PDLC and porous silicon (PS) $[3,5,6]$. From the photoluminescence and absorption spectra of PDLC, spanning the 
wavelength interval $290-850 \mathrm{~nm}$ and $1.5-4.5 \mathrm{eV}$, respectively, the energy features for a number of electronic processes are determined. These suggest, like PS, the possibility of technological applications in opto-electronic devices.

A gradient structural pattern classification of the SFM porosity, by means of the computational gradient pattern analysis (GPA), GPA-flyby environment, is performed on PDLC samples. The results show many structural similarities, as gradient asymmetries and topological entropies compared to real PS samples.

\section{Experimental details}

The PDLC thin films were obtained on (100) silicon substrate by DC low energy $(200 \mathrm{~W})$ magnetron sputtering of a graphite target with the discharge produced in a gas mixture of $10 \% \mathrm{H}_{2}$ and $90 \%$ argon and stored in atmosphere environment. The roughness (Rms) for PDLC is around $5 \mathrm{~nm}$, and it does not change with the scanning area [2]. The PS samples were produced by etching p-type c-Si wafers of crystal orientation (100) and resistivity $1-$ $30 \Omega \mathrm{cm}$ electrochemically in $40 \% \mathrm{HF}$ :ethanol $1: 1$. The electrolytic cell as well as the etching procedure are described in Refs. $[5,6]$. The experimental absorption and reflection spectra were obtained by a homemade reflection-transmission spectrometer. The experimental photoacoustic spectroscopy (PAS) apparatus consists of a high-pressure $1000 \mathrm{~W}$ xenon arc lamp (Osram), a chopper (HMS Elektronik, model 220A) and a monochromator (Sciencetech, model 9010). The ellipsometric data were recorded at room temperature with variable angle of incidence spectroscopic ellipsometry (J.A. Woollam, Inc. Co.) in the photon energy range $0.75-6.5 \mathrm{eV}$ [7]. The photoluminescence measurements for two excitation wavelengths were performed following standard procedures [5]. The surface of the samples was studied using a Shimadzu scanning force microscopy in tapping mode.

To determine the coefficient of thermal diffusivities of PDLC, PS and crystalline silicon c-Si, a $300 \mathrm{~mW}$ Melles Griot argon laser was used as light source. The monochromatic light beam was modulated with a chopper; model SR540 of Stanford Research Systems (SRS). The cell used allows front and rear illuminations. The signal produced in the cell was detected with a Seinheiser microphone, amplified with the Lock-in of the SRS, recorded and interpreted by a computer program. The amplitude and phase of the PA signals of the PDLC, PS and c-Si were measured at different frequencies of modulation. These measurements were carried out with rear illumination, first with the film face receiving the light and afterwards with the substrate face illuminated. In both cases the thermal diffusivity coefficient found no significant differences.

\section{Modeling and numerical results for GPA}

Recently, the characterization of irregular structures in PS SFM images has been studied using the gradient pattern analysis (GPA) method [6,8-10]. GPA is an innovative technique, which characterizes the formation and evolution of extend patterns based on the spatial correlations between large and small amplitude fluctuations of the structure represented as a gradient field [11,12].

The application of GPA for PS SFM images analysis was performed on images of three typical PS samples distinguished by different absorption energy levels and porosity aspect ratios (low, intermediate and high roughness) [6].

The structure formation GPA models can be discrete or continuous where the discrete models demand to simulate automata universal aspects of the problem. Continuous models generally use differential equations, with stochastic component, where the main formation process obeys the general physical aspects of the problem. The continuous model used in this work is the (Kardar-Parisi-Zhang) KPZ equation. [13]:

The KPZ equation is given by the following expression

$\frac{\partial h(x, t)}{\partial t}=v \nabla^{2} h+\frac{\lambda}{2}(\nabla h)^{2}+\eta(x, t)$,

where $h(x, t)$ is the height (position $x$, time $t$ ), $v$ is the parameter of surface tension, $\lambda$ is the parameter of lateral growth and $\eta(x, t)$ is the noise term. A result from KPZ 2D simulation, solved by means of the FTCS (forward time centered space) method [8], is shown in Fig. 1 where, the spatio-temporal scales $(L \times L$, with $0.1 \mu \mathrm{m}$ per pixel) are compatible to the real PS scales showing similarities with Fig. 2.

Figs. 2 and 3 show a SFM image of PS sample produced with 60 min, etching time and the SFM image of the PDLC sample used in this work, respectively. X-ray photoelectron spectroscopy (XPS) analysis has been performed on this PDLC film showing dominant $\mathrm{C}-\mathrm{H}$ bond and some oxygen concentrations [2].

The KPZ model presented results compatible with the irregular surfaces observed in the SFM samples of PS and PDLC when their constitutive parameters (surface

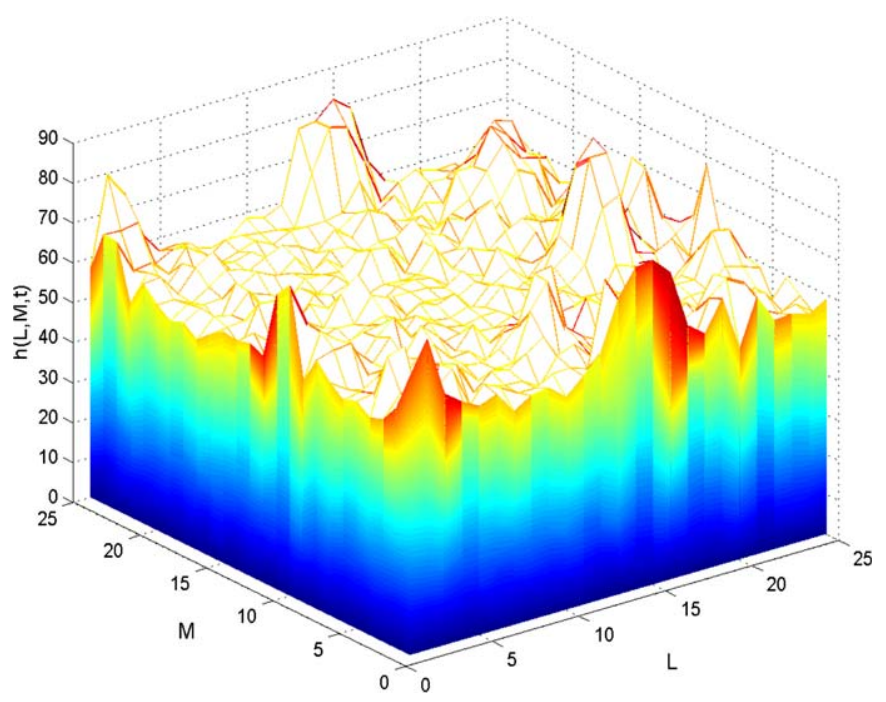

Fig. 1. An example of simulation by KPZ 2D model. 


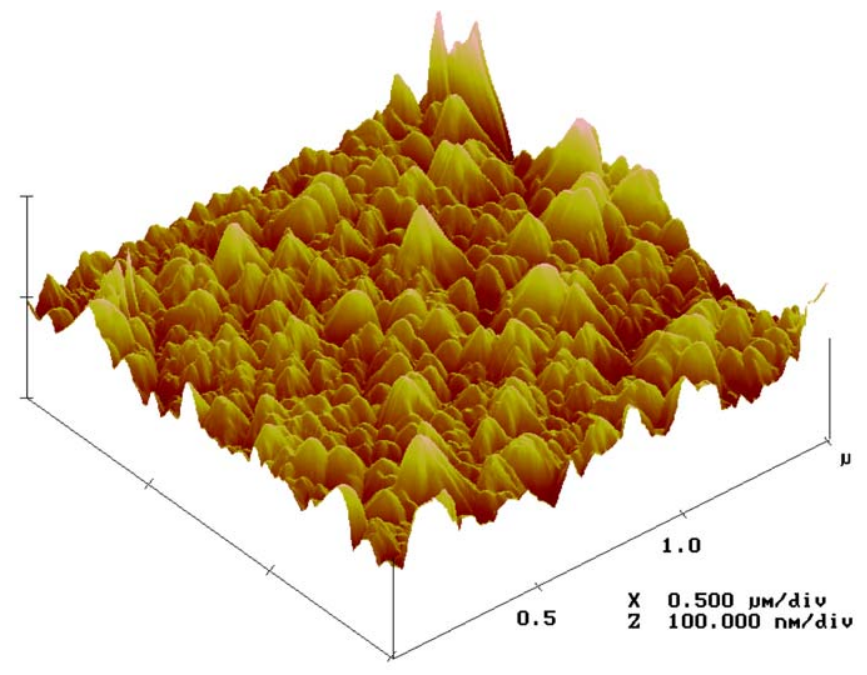

Fig. 2. Scanning force microcopy image of the porous silicon surface.

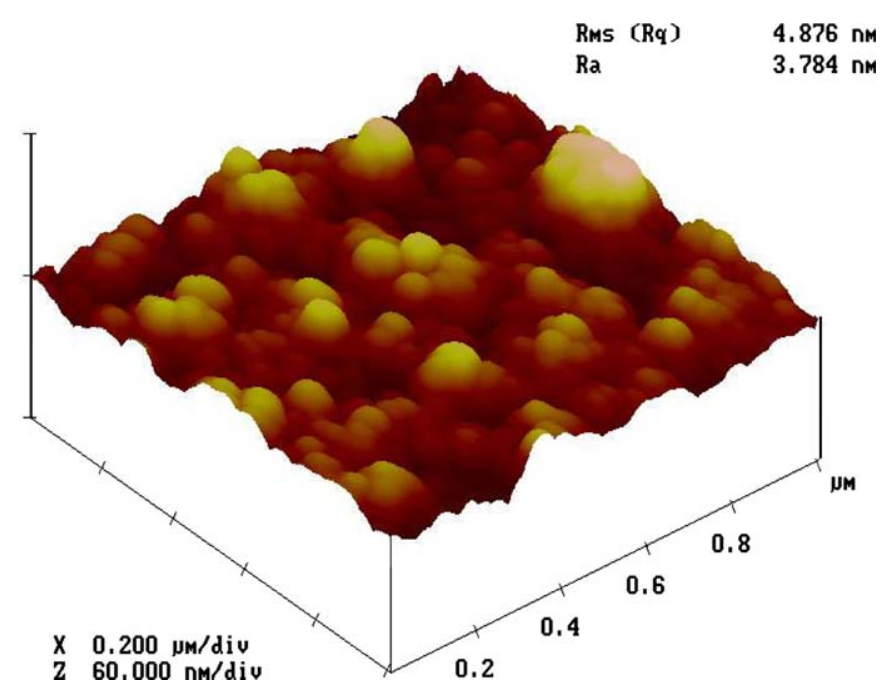

Fig. 3. Scanning force microcopy image of the PDLC surface. tension, noise term and lateral growth) are in the following ranges: $-0.3 \leqslant v \leqslant-0.1 ;-1 \leqslant \sigma \leqslant-0.5$ and $\lambda$ without restriction. This analysis has been adapted in order to operate in an OpenGL flyby environment (the StrFB code) [9], whose application gives the numerical characterization of the structure during the flyby in real time (Fig. 4).

\section{Results}

The dielectric function was calculated in the momentum representation, which requires matrix elements of the momentum, $p$, between occupied and unoccupied eigenstates. To be specific the imaginary part of the dielectric function, $\varepsilon_{2}(\omega)=\operatorname{Im} \varepsilon(q=0, \varepsilon)$, was calculated from [4]

$$
\begin{aligned}
\varepsilon_{2}^{i j}(\omega)= & \frac{4 \pi^{2} e^{2}}{\Omega m^{2} \omega^{2}} \sum_{\mathbf{k} n n^{\prime} \sigma}\left\langle\mathbf{k} n \sigma\left|p_{i}\right| \mathbf{k} n^{\prime} \sigma\right\rangle\left\langle\mathbf{k} n^{\prime} \sigma\left|p_{j}\right| \mathbf{k} n \sigma\right\rangle \\
& \times f_{\mathbf{k} n}\left(1-f_{\mathbf{k} n^{\prime}}\right) \delta\left(e_{\mathbf{k} n^{\prime}}-e_{\mathbf{k} n}-\hbar \omega\right) .
\end{aligned}
$$

The imaginary part of the dielectric function, $\varepsilon_{2}(\omega)$, in the long wavelength limit have been obtained directly from the electronic structure, using the joint density-of-states and the optical matrix overlap, see Ref. [4]. In Eq. (2), $e$ is the electron charge, $m$ its mass, $\Omega$ is the crystal volume and $f_{\mathbf{k} n}$ is the Fermi distribution. Moreover, $|\mathbf{k} n \sigma\rangle$ is the crystal wave function corresponding to the $n$th eigenvalue with crystal momentum $\mathbf{k}$ and spin $\sigma$. The summation over the Brillouin-zone in Eq. (2) is calculated using the tetrahedron interpolation with a $k$-mesh consisting of about 450 uniformly distributed $k$-points. The matrix elements, eigenvalues and eigenvectors are calculated in the irreducible part of the Brillouin-zone. Finally, the real part of the dielectric function, $\varepsilon_{1}(\omega)$, is obtained from $\varepsilon_{2}(\omega)$ using the Kramers-Kronig transformation

$$
\begin{aligned}
\varepsilon_{1}(\omega) & \equiv \operatorname{Re}(\varepsilon(q=0, \omega)) \\
& =1+\frac{1}{\pi} \int_{0}^{\infty} \mathrm{d} \omega^{\prime} \varepsilon_{2}\left(\omega^{\prime}\right)\left(\frac{1}{\omega^{\prime}-\omega}+\frac{1}{\omega^{\prime}+\omega}\right) .
\end{aligned}
$$

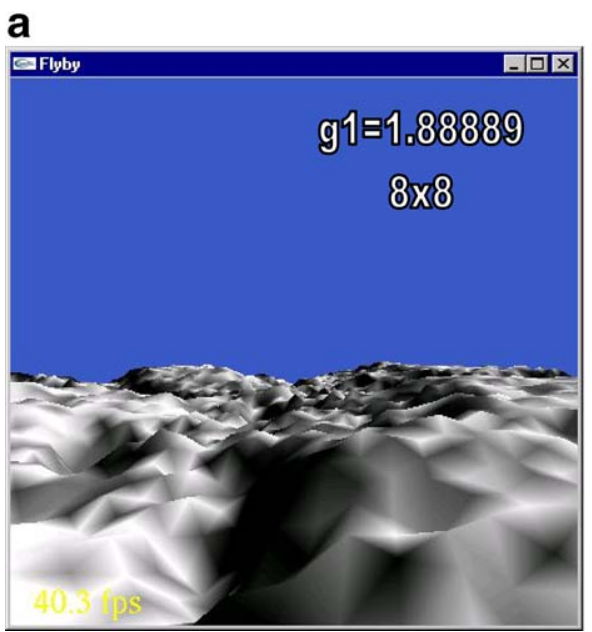

b

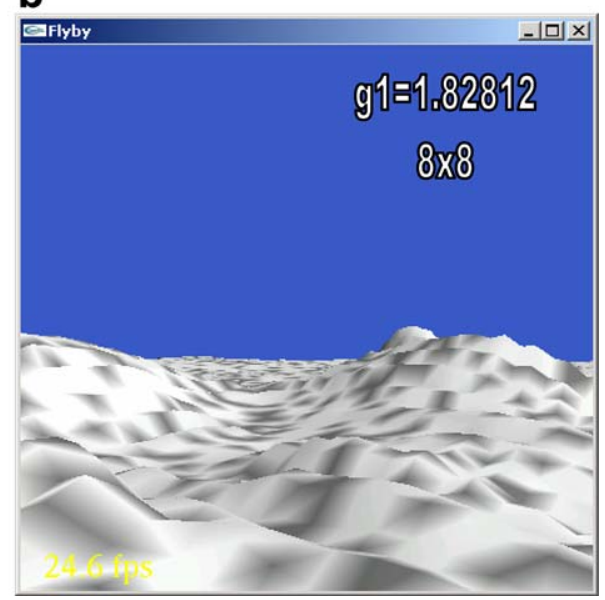

Fig. 4. Flyby using the StrFB code, computing the level of correspondent local asymmetry in an area of $8 \times 8$ : (a) flyby snapshot of SFM structural pattern of a porous silicon; (b) flyby snapshot SFM structural pattern of a porous diamond-like carbon. 


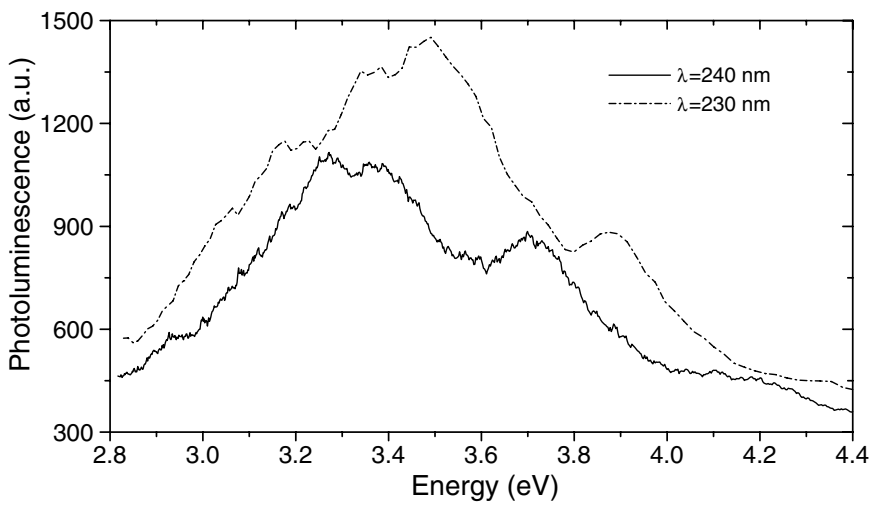

Fig. 5. Photoluminescence of PDLC.

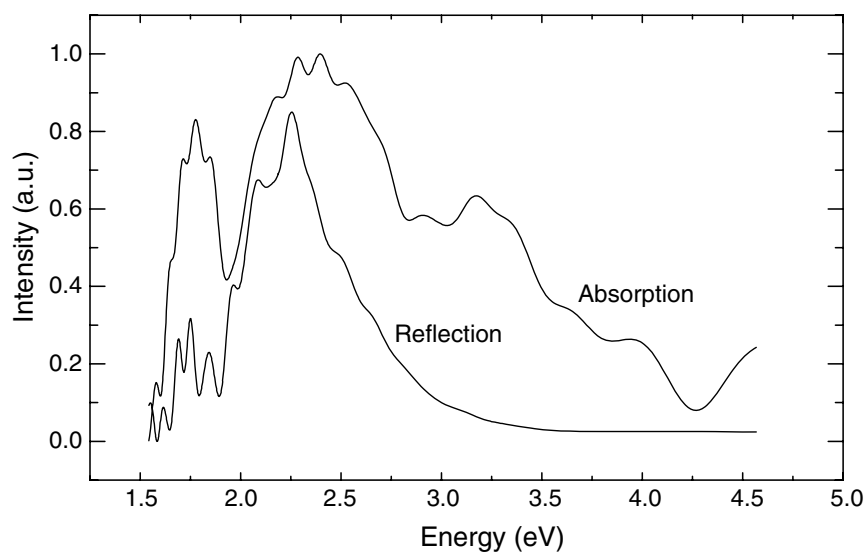

Fig. 6. Absorption and reflection of PDLC.

Fig. 5 presents the photoluminescence measurement of a PDLC sample, where dotted and full lines are related to the excitation wavelengths of $230 \mathrm{~nm}$ and $240 \mathrm{~nm}$, respectively. The photoluminescence was observed just in the interval $280-440 \mathrm{~nm}$. Such PL depends on the PDLC homogeneity.

Fig. 6 shows the absorption and reflection spectra in the energy range $1.5-4.5 \mathrm{eV}$. These spectra reveal complex structures in contrast to those obtained from ordinary semiconductor materials with one fundamental band-gap, for which a well-defined absorption edge is observed. Such complexity has also been found in porous silicon and it is originated from the surface roughness as shown in Fig. 7.

Fig. 8 presents the photoluminescence measurement of a PS sample. Table 1 shows the 16 features observed in the absorption spectrum of the PDLC and also those observed to PS in Figs. 6 and 7, respectively. It is worth noting that in the energy region studied here, the absorption measurements show some similarities with the energy structure of

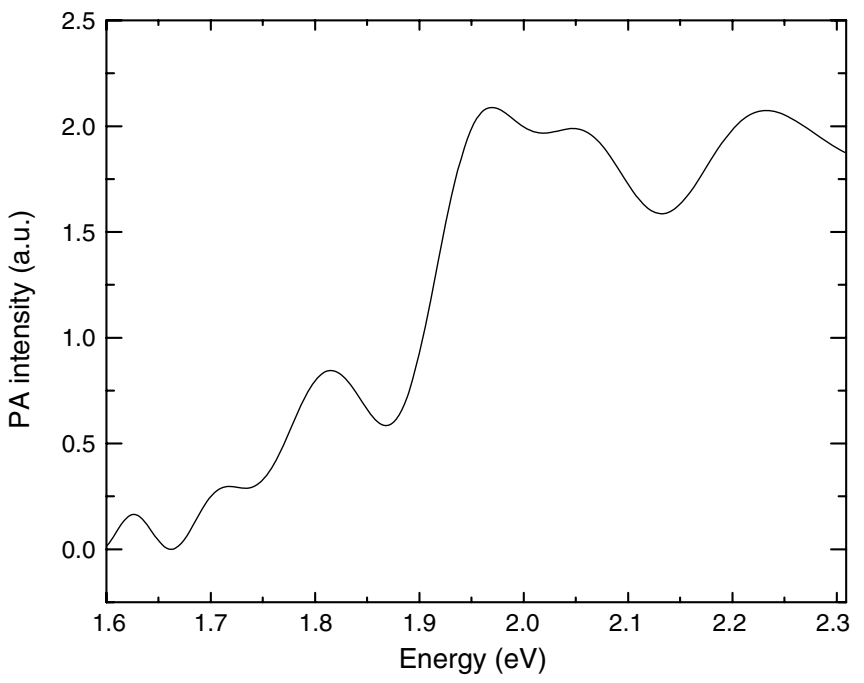

Fig. 7. Absorption of PS.

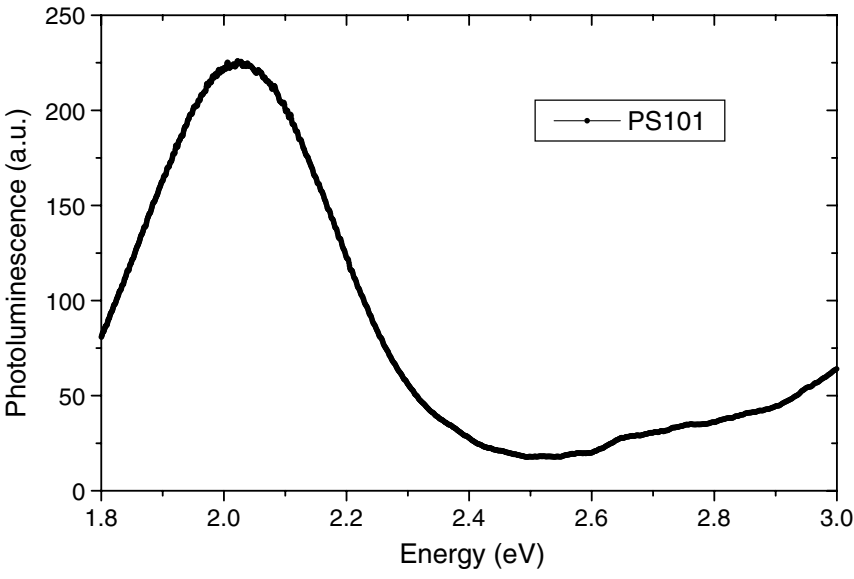

Fig. 8. Photoluminescence of PS.

PS investigated with photoluminescence excitation (PLE) and photoacoustic spectroscopy (PAS) for energies below $2.2 \mathrm{eV}[5]$.

From the measured dielectric functions for PDLC a low real dielectric constant of about 4.5 at $0.8 \mathrm{eV}$ was found whereas the calculated and measured values of $e_{1}(0)$ for BDLC are 5.5 and 5.7, respectively. The former result

Table 2

Morphological aspects for the best surfaces generated by the $2 \mathrm{D} \mathrm{KPZ}$ model and PS and PDLC for scale of $25 \times 25$

\begin{tabular}{llll}
\hline & 2D KPZ & PS & PDLC \\
\hline Gradient pattern $\left(g_{1}^{\mathrm{a}}\right)$ & $1.970 \pm 0.001$ & $1.970 \pm 0.004$ & $1.970 \pm 0.005$
\end{tabular}

Table 1

Values of the absorption energy onsets for PDLC and PS

\begin{tabular}{|c|c|c|c|c|c|c|c|c|c|c|c|c|c|c|c|c|}
\hline PDLC-AB (eV) & 1.56 & 1.63 & 1.69 & 1.75 & 1.84 & 2.02 & 2.13 & 2.25 & 2.37 & 2.49 & 2.68 & 2.87 & 3.11 & 3.31 & 3.60 & 3.90 \\
\hline PS-PAS (eV) & 1.55 & 1.62 & 1.67 & 1.75 & 1.86 & 2.02 & 2.14 & - & - & - & - & - & - & - & - & - \\
\hline
\end{tabular}


indicates a low $e_{1}(0)$ for PDLC as pointed out by Sakaue et al. [14].

This paper also showed asymmetry level for the KPZ 2D model compatible with the surfaces observed in the PS and PDLC samples (Table 2).

\section{Conclusions}

In this work the complex structure of the band-gap energies of the PDLC was obtained through the transmission and reflection techniques. Such complex structure exhibits a total of 16 energies onsets in the energy range 1.5$4.5 \mathrm{eV}$. This is by itself an usual richness in structures in comparison to cases where semiconductor materials with ordinary band structures have been studied, and it shows similarities with PS and the two broad peak energies as observed in CVD diamond films [15]. This suggests, like PS, that PDLC also has a potential for applications in optic-electronic device structures. The photoluminescence of the PDLC is also shown. The X-ray photoelectron spectroscopy (XPS) analysis on this PDLC film has shown dominant $\mathrm{C}-\mathrm{H}$ bond and some $\mathrm{O}$ concentrations [2]. This occurrence probably leads the PDLC to have a very lower thermal diffusivity when compared to bulk diamond but only half the value of silicon and higher value compared to PS. Both PDLC and PS use silicon as substrate. PS has treated electrochemically in 40\% HF:ethanol 1:1. Moreover due to a very low roughness and thickness of the PDLC thin film compared to silicon substrate we treat the thermal diffusivity as an effective mean value. This has been done for porous silicon with very good results [16-18].

The PDLC effective thermal diffusivity result was found $\alpha=0.48 \mathrm{~cm}^{2} / \mathrm{s}$, a value, which is about 40 times less for diamond and half of the crystalline silicon, $\alpha=0.9 \mathrm{~cm}^{2} / \mathrm{s}$, and about 10 times for PS, i.e., $\alpha=0.045 \mathrm{~cm}^{2} / \mathrm{s}$ [16]. This last value may be compared to the other effective values for PS, $\alpha=0.047 \mathrm{~cm}^{2} / \mathrm{s}$ and $\alpha=0.053 \mathrm{~cm}^{2} / \mathrm{s}$ found by CruzOrea et al. [17] and Calderón et al. [18], respectively, with open photoacoustic cell method.

For the dielectric functions we have performed the spectroscopic ellipsometry. The result has shown the tendency of the PDLC dielectric constant becomes lower when compared to the BDLC. The calculated and measured values of $e_{1}(0)$ showed a very good agreement. The KPZ model has shown structurality results, for $2.5 \mu \mathrm{m} \times 2.5 \mu \mathrm{m}$ scales, compatible with the irregular surfaces observed in the SFM samples of PS and PDLC.

\section{Acknowledgements}

This work was financially supported in part by the Brazilian Agencies CNPq, REMAN/CNPq, FAPESB (Bahia), the Swedish Foundation for International Cooperation in Research and Higher Education (STINT) and Swedish Research Council (VR). Discussions with L. Vieira Santos and V.J. Trava-Airoldi are acknowledged. M.P.M.A.B acknowledges Nandamudi L. Vijaykumar for valuable English corrections.

\section{References}

[1] L.V. Santos, V.J. Trava-Airoldi, K. Iha, E.J. Corat, M.C. Salvadori, Diam. Relat. Mater. 10 (2001) 1049

[2] L.V. Santos, V.J. Trava-Airoldi, K. Iha, M. Massi, R. Prioli, R. Landers, Diam. Relat. Mater. 11 (2002) 1135.

[3] L.T. Canham (Ed.), Properties of Porous Silicon, EMIS Data Review Series, Number 181 NSPEC, London, 1997 (Chapters 6, 7 and 10).

[4] C. Persson, R. Ahuja, A. Ferreira da Silva, B. Johansson, J. Phys. Condens. Matter 13 (2001) 8945

[5] R.G. Egeberg, E. Veje, A. Ferreira da Silva, I. Pepe, A. Santos Alves, J. Porous Mater. 7 (2000) 173.

[6] A. Ferreira da Silva, R.R. Rosa, L.S. Roman, E. Veje, I. Pepe, Solid State Commun. 113 (2000) 703.

[7] R. Ahuja, H. Arwin, A. Ferreira da Silva, C. Persson, J.M. OsórioGuillon, J. Souza de Almeida, C. Moyses Araújo, E. Veje, N. Veissid, C.Y. An, I. Pepe, B. Johansson, J. Appl. Phys. 92 (2002) 7219.

[8] M.P.M.A. Baroni, R.R. Rosa, A. Ferreira da Silva, I. Pepe, L.S. Roman, F.M. Ramos, R. Ahuja, C. Persson, E. Veje, Microelectron. J. 33 (4) (2006) 290.

[9] R.R. Rosa, A. Ferreira da Silva, R.C. Brito, L.S. Roman, M.P.M.A Baroni, F.M. Ramos, R. Ahuja, C. Persson, Phys. Status Solidi C 1 (S2) (2004) S277.

[10] A.F. da Silva, R.R. Rosa, P.W.A. Machado, F.M. Ramos, C.R. Neto, L.S. Roman, E. Veje, Physica A - Statist. Mech. Appl. 283 (12) (2000) 223 .

[11] R.R. Rosa, A.S. Sharma, J.A. Valdivia, Int. J. Mod. Phys. C 10 (1) (1999) 147.

[12] R.R. Rosa, M.R. Campos, F.M. Ramos, N.L. Vijaykumar, Braz. J. Phys. 33 (3) (2003) 605.

[13] M. Kardar, G. Parisi, Yi-C. Zhang, Phys. Rev. Lett. 56 (1986) 889.

[14] H. Sakaue, N. Yoshimura, S. Shingubara, T. Takahagi, Appl. Phys. Lett. 83 (2003) 2226.

[15] M.C. Rossi, S. Salvatori, F. Galluzzi, Diam. Relat. Mater. 6 (1997) 712 .

[16] A. Ferreira da Silva, T.S. Silva, O. Nakamura, M.M.F. Aguiar Neto, I. Pepe, L.S. Roman, E. Veje, Mater. Res. 4 (2001) 23.

[17] A. Cruz-Orea, I. Delgadillo, H. Vargas, A. Gudino-Martinez, E. Marin, C. Vazquez-Lopez, A. Calderon, J.J. Alvarado-Gil, J. Appl. Phys. 79 (1996) 8951.

[18] A. Calderón, J.J. Alvarado-Gil, Y.G. Gurevich, A. Cruz-Orea, I. Delgadillo, H. Vargas, L.C.M. Miranda, Phys. Rev. Lett. 79 (1997) 5022 . 